\title{
Applicable ultrafast all-optical switching by soliton self-trapping in high index contrast dual-core fiber
}

\author{
M. Longobucco $^{a, b}$, P. Stajanča ${ }^{c}$, L. Čurilla ${ }^{d}$, R. Buczyński $^{a, b}$, I. Bugár $^{a, e}$ \\ ${ }^{a}$ Department of Glass, Lukasiewicz Research Network - Institute of Electronic Materials Technology, \\ Wólczyńska 133, 01-919 Warsaw, Poland \\ ${ }^{b}$ Faculty of Physics, University of Warsaw, Pasteura 5, 02-093 Warsaw, Poland \\ ${ }^{c}$ BAM Bundesanstalt für Materialforschung und -prüfung, Unter den Eichen 87, 12205 Berlin, Germany \\ ${ }^{d}$ Optics11, De Boelelaan 1081, 1081 HV Amsterdam, Netherlands \\ ${ }^{e}$ International Laser Centre, Ilkovičova 3, 84104 Bratislava, Slovakia
}

\begin{abstract}
Improvement potential of ultrafast all-optical switching by soliton self-trapping using all-solid dual-core fibers with high index contrast was analyzed numerically. Study of the femtosecond nonlinear propagation was performed based on the coupled generalized nonlinear Schrödinger equations considering three different fiber architectures: homogeneous cladding all-solid, photonic crystal air-glass and photonic crystal all-solid. The structure geometries of the all three alternatives were optimized in order to support high-contrast switching performance in C-band considering $100 \mathrm{fs}$ level pulse widths. Comparing the three different structural alternatives, the lowest switching energies at common excitation parameters $(1700 \mathrm{~nm}$ and $70 \mathrm{fs}$ pulses) were predicted for the homogeneous cladding dual-core structure. The further optimization of the excitation wavelength and pulse width resulted in lower switching energies at simultaneous improvement of the switching contrasts at combination of $1500 \mathrm{~nm}, 75 \mathrm{fs}$ pulses and at $43 \mathrm{~mm}$ fiber length. The spectral aspect in this optimized case expresses broadband and uniform switching character spanning over $200 \mathrm{~nm}$ and exceeding $30 \mathrm{~dB}$ contrast at more frequency channels.
\end{abstract}

\section{Introduction}

The all-optical signal processing is a hot topic nowadays, in the era of increasing demand of speedup of the data transfer rates. High-speed optical fiber-based long distance data transport needs compatible devices in order to switch [1], route [2] or buffer [3] the signal in optical way. The standard fiber-based solution of ultrafast all-optical switching is a Sagnac interferometer or nonlinear optical loop mirror, demonstrated among others already for quantum computation tasks [4]. However, such device requires at least meter-level fiber length and additional couplers in order to separate the signal and switching pulses. Therefore, a simple dual-core fiber-based nonlinear directional coupler approach has been constantly studied in the last decades in order to provide more compact and low energy requirement all-optical switching alternative [5-7]. After the first experiments of ultrafast pulse switching in dual-core fiber, which uncovered its limitations [8], the attention of nonlinear fiber community was drawn to the solitonic approach [9]. Large number of theoretical works has been dedicated to this topic, analyzing the potential of solitonic pulses to mitigate the different pulse distortion processes and to give higher switching contrast possibilities in various structures [10,11]. Modifications of the generalized nonlinear Schrödinger equation (GNLSE) have been used and different approaches have been suggested theoretically in order to enhance the waveguide nonlinearity by highly nonlinear glass or by infiltrating liquids or liquid crystals into the fiber core $[12,13]$. Many of the mentioned works use photonic crystal fibers (PCF), which allows wide tailoring possibilities of the fiber dispersion, coupling and nonlinear characteristics [1-3,12,13].

Despite the optimistic theoretical predictions, only few experimental papers have been published. Those up to date have not overcome the $15 \mathrm{~dB}$ switching contrast already achieved three decades ago in the case of a classical dual-core fiber excited by femtosecond pulses in the normal dispersion region [8]. In the case of dual-core PCF excited in anomalous dispersion region, our group achieved this level of switching contrast, however, only in a limited spectral region of the nonlinearly broadened spectra [14]. Simultaneously, a 
numerical model based on the coupled GNLSE has been developed, which allowed us to obtain comparable numerical results to the experimental ones in the case of different types of dual-core PCFs [14,15]. Our recent focus is the design and development of a dual-core fiber, which will support high contrast all-optical switching. Using the verified numerical model, a new concept of switchable self-trapping of high-order solitons in the both fiber cores has been elaborated [16]. The introductory numerical paper about this approach was followed by an experimental work on optimized air-glass dual-core PCF, when its output characteristics under excitation by tunable femtosecond source in range of $1500-1800 \mathrm{~nm}$ were measured [15]. The results confirmed our predictions about the possibility of all-optical switching at sub-nanojoule pulse energy level thanks to the used highly nonlinear lead-silicate glass. On the other side, the demonstration of the self-trapped solitonic switching failed due to the fibers dual-core asymmetry. According to our long time experience, the required level of dual-core symmetry is hardly attainable with highly nonlinear glasses using the standard air-glass PCF architecture.

However, a new technology emerged recently, which can address our novel ultrafast all-optical fiber-based switching concept; namely, the all-solid PCF approach. The combination of two thermally matched glasses in the fiber structure is a matured fabrication method for us and already brought several new achievements for single-core optical fibers [17,18]. All-solid PCF technology is reported by other authors as well, mainly in area of fiber laser development $[19,20]$. The substitution of the air holes in the fiber structure with low index glass rods undoubtedly improves the integrability potential of such waveguides to standard fiber communication systems. Moreover, dual-core all-solid PCF has been already manufactured and utilized for switchable multi-wavelength fiber laser [21]. Motivated by this technology background, we started a new series of fiber geometry optimization implementing the combination of the previously used highly nonlinear glass with a thermally matched low index borosilicate glass [18]. The recent extensive simulation work on the air-glass PCF, all-solid PCFs and simple all-solid dual-core fibers allowed us to acquire deeper understanding of the conditions which support self-trapped soliton switching.

In our first publication, where the soliton self-trapping idea was introduced, the key optimization condition of an air-glass PCF was the comparable value between the coupling length $L_{\mathrm{C}}$ and the period of the highorder solitonic propagation $z_{0}$. Our work was motivated by an earlier theoretical paper of Wang and Wang, where the ground of the discrepancy between the theoretical predictions and the experimental demonstrations of the dual-core fiber solitonic switching has been analyzed [6]. They showed that the ratio $z_{0} / L_{\mathrm{C}}$ determines the character of the switching performance. We followed this concept and showed that an air-glass PCF optimized to have $z_{0} / L_{\mathrm{C}} \cong 6$ gives the possibility to have switching based on soliton self-trapping [16]. Our numerical studies revealed that such theoretical considerations are valid, because different types of dual-core fiber structures can satisfy the above-mentioned condition and therefore exhibit high-contrast switching [22]. One can refine the key optimization condition taking into consideration the targeted pulse energy level and focusing on the soliton compression distance $z_{\text {comp }}$, rather than on the soliton period. However, we recognized, that moderate level of soliton order is required in addition to the comparable soliton compression distance and coupling length. Soliton order higher than 1 is necessary because only high-order solitons exhibit the soliton compression phenomenon [23]. On the other hand, very high- order solitons (above 10) do not support the effective self-trapping process due to splitting into numerous fundamental solitonic components during the soliton fission process [24]. Both the soliton order $N$ and the soliton compression distance $z_{\text {comp }}$ are dependent on the parameters of the fiber and of the exciting pulses according to the following relations [23]:

$$
N^{2}=\frac{\gamma P_{0} T_{0}^{2}}{\left|\beta_{2}\right|}
$$

In the first one above, $P_{0}$ is the pulse peak power, $\gamma$ is the nonlinear coefficient, $T_{0}$ is the pulse duration and $\beta_{2}$ is the group velocity dispersion. When $N>2$, a high-order soliton is generated and periodically recovers 
after a soliton period $z_{0}$, defined as a function of pulse duration and group velocity dispersion expressed by relation [23]

$$
z_{0}=\frac{\pi T_{0}^{2}}{2\left|\beta_{2}\right|}
$$

The soliton compression distance is a non-trivial function of the above-mentioned quantities. However, in the case of $N>10$, it is possible to approximate it by equation [23]

$$
z_{\text {comp }}=\left(\frac{0.32}{N}+\frac{1.1}{N^{2}}\right) z_{0}
$$

By inserting of the equations (1) and (2) for $N$ and $z_{0}$, into the soliton compression length equation (3), it is possible to estimate the range of input pulse power, which supports the self-trapped solitonic switching, when condition $L_{c} \approx z_{\text {comp }}$ is satisfied. The equation results in:

$$
z_{\text {comp }}=0.32 \cdot \frac{\pi T_{0}}{2 \sqrt{\gamma P_{0}\left|\beta_{2}\right|}}+1.1 \cdot \frac{\pi}{2 \gamma P_{0}}
$$

By fixing the input pulse parameters $P_{0}$ and $T_{0}$, the soliton compression distance $z_{\text {comp }}$ and taking into a specific fiber design and excitation wavelength (therefore, by setting a value for the parameters $\gamma$ and $\beta_{2}$ ), an estimated value of $P_{0}$ can be calculated.

We will focus standardly on soliton orders lower than 10 . Nevertheless, we can use relation (3) for the $z_{\text {comp }}$ as a qualitative guide for the understanding of the soliton compression principles. In the frame of numerical analysis of nonlinear propagation in different types of dual-core fiber structures, it is always necessary to identify at first the pulse energy level, which fulfill the condition of comparable $z_{\text {comp }}$ and $L_{\mathrm{C}}$. However, these structures can differ from each other from the point of view of nonlinearity and anomalous dispersion at the identified energy level. Therefore, the complex excitation and fiber parameters in each case can exhibit different level of soliton order, according to equation (1). One of our recent works was focused on the selftrapped soliton switching concept in a simple dual-core fiber structure at different soliton orders, by mutual change of the excitation wavelength and the pulse width. In this case the optimal value of $N$ was at level of 2-3 [22].

In this paper, improved performance of all-optical switching based on self-trapping of high-order solitons in new types of dual-core (DC) fibers is studied numerically. The introduced switching concept, previously elaborated for the case of air-glass DC PCF, is now implemented for fibers which are made of a special pair of multicomponent glasses expressing high refraction index contrast. We will present the optimization process of a fiber structure with an aim of achieving proper anomalous dispersion profile in the C-band. The optimization is performed by means of numerical simulation of fiber's linear optical properties. Then the self-trapped soliton switching is analyzed and compared between air-glass PCF, all-solid PCF and simple allsolid DC structures. Finally, the best results in the case of simple DC fiber under optimized excitation conditions are presented. These results exhibit better switching performance than in the case of the original air-glass DC PCF approach. Thanks to this extensive study, the key conditions resulting in maximal switching contrast are identified and a realistic fiber structure is suggested both from point of view of technology and low loss excitability.

\section{Numerical methods}

The detailed description of the numerical methods used for the optimization of the fiber geometry and for the analysis of the nonlinear propagation was published already elsewhere [22]. Now we just summarize shortly the most important aspects and working procedures. The structure of the DC fiber was initially optimized by the proper choice of material for the future technology process. The selection of the two glasses for the cores 
and for the cladding was a result of an extensive research, including synthesis of a complementary glass to the highly nonlinear lead-bismuth-gallium-silicate (PBG-08) glass, already used in the case of the air-glass DC PCF structure $[15,16]$. The optimization process resulted in a glass composition labeled as UV-710 allowing air-like behavior of the areas filled with it. In combination with PBG-08 glass, it provided refraction index contrast around 0.4 in a wide spectral region (1400 - $2000 \mathrm{~nm})$ [18]. At the beginning, a periodic hexagonal lattice of hexagonal low index glass rods was considered (Fig. 1a), enabling the study of higher low index area fill factors than the cylindrical rods.
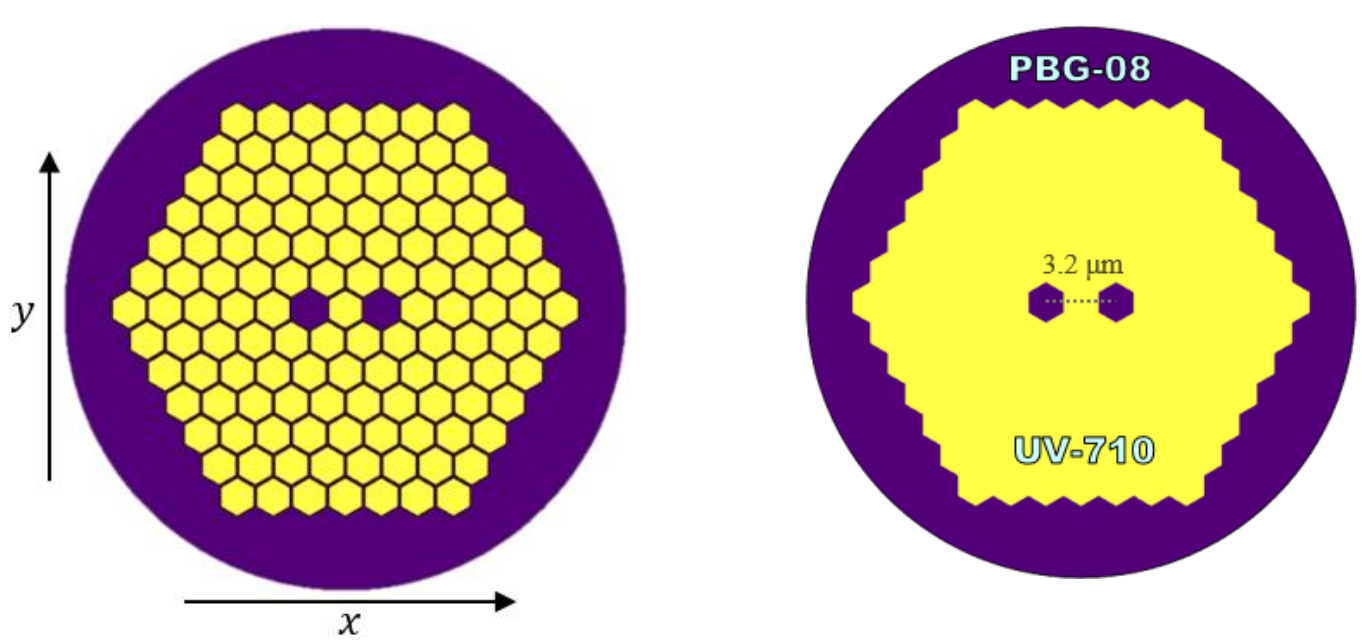

Fig. 1 Hexagonal array of hexagonal rods type dual-core all-solid PCF fiber with lattice with (left) high and (right) maximal low index fill factor of the cladding. In the latter case, the two cores and the low index microrods have the same size.

In the next step, the new structure was studied from the point of view of optical field propagation characteristics in linear regime. The commercial Mode Solution software from Lumerical was used to calculate the spectral dependences of the field mode profile, the effective refractive index and the waveguide losses for each fundamental mode. The all relevant quantities like effective mode area, dispersion, coupling coefficient, losses were acquired in spectral window between 0.3 and $4.1 \mu \mathrm{m}$, which sufficiently covers our region of interest. Both mode identification and dispersion characteristics calculation were initially performed for an artificial single-core structure, which was obtained by extending the periodical lattice for the area of one of the cores. In the second step, the more complex dual-core structure was used to determine the coupling coefficient between the two single core modes based on the overlap integrals [23].

Our previous theoretical work in the case of air-glass PCF [16] and all-solid simple DCF [22] already showed, that the linear optical characteristics in the studied range of structural parameters and wavelengths are very similar for the both main polarization directions. Therefore, in this paper, we will focus only on the $\mathrm{X}$ polarized field, which is parallel with the connecting line of the two cores, both in the case of linear and nonlinear propagation characteristics.

The structure of a dual-core fiber was optimized from the point of view of geometry in order to ensure as high as possible anomalous dispersion in the area of the optical communication bands (1400 nm - $1700 \mathrm{~nm})$. Further aspects of the optimization process were to minimize the third order dispersion (TOD) effect and to achieve sufficient nonlinearity for picojoule pulses with duration at level of $100 \mathrm{fs}$. Standardly, there is a trade-off between the dispersion and nonlinearity aspects, because the larger mode area often supports higher anomalous dispersion. Moreover, it is necessary to keep the coupling length at the $\mathrm{mm}-\mathrm{cm}$ area, because, in order to preserve the device compactness, it should be balanced with the $z_{\text {comp }}$, as explained in the introduction. The coupling length increases with increasing overall structural dimension at unchanged fiber architecture. 
The single core fiber characteristics together with the identified coupling coefficients are then used in the nonlinear simulation, where the coupled generalized nonlinear Schrödinger equations (CGNSE) are solved numerically [14]. Both experimentally determined instantaneous Kerr and delayed Raman response of the guiding PBG-08 glass are included into the material nonlinear response function. The equations were solved numerically by the split-step Fourier method with 40000 longitudinal steps; this value was already optimized during the previous air-glass PCF study [16]. After every 200 calculation step, the field arrays were saved and then used to generate the output propagation maps; this means that the whole propagation distance is divided into 200 points. This approach represents a good compromise between the calculation time and the resolution of the propagation distance. For the numerical simulations, the input pulse shape is represented by the $\operatorname{sech}^{2}$ function, which is good approximation for ultrafast oscillators with pulse widths at the level of 100 fs.

\section{Optimization of the linear optical characteristics}

During the optimization process, hexagonal structure of DC PCF with low index hexagons in a high index matrix was taken into consideration, with uniform dimensions and period (or pitch, $\Lambda$ ) of the hexagons (Fig. 1a). The two fiber cores are composed of two defects in the hexagonal structure separated from each other by one low index hexagon. The place of the missing hexagons is filled with the high index glass, so the structure is all-solid without any air containing areas. The optimization was focused on the requirement of as high as possible anomalous dispersion in order to keep $N$ at a moderate level, according to equation (1). Further requirement was the flatness of the dispersion profile in the C-band, in order to eliminate the disadvantageous soliton fission and dispersive wave generation effect. The third aspect was to keep the core diameter below $2 \mu \mathrm{m}$, which ensures highly nonlinear interaction of the femtosecond pulses even in the range of picojoule pulse energies. The optimization process was realized by systematic change of the $\Lambda / r$ at constant pitch and of the pitch at constant $\Lambda / r$, where $r$ is the outer radius of the hexagonal rods. Different constant values of $\Lambda$ and $\Lambda / r$ were explored in ranges $1.3-1.8 \mu \mathrm{m}$ and $2-3$, respectively.
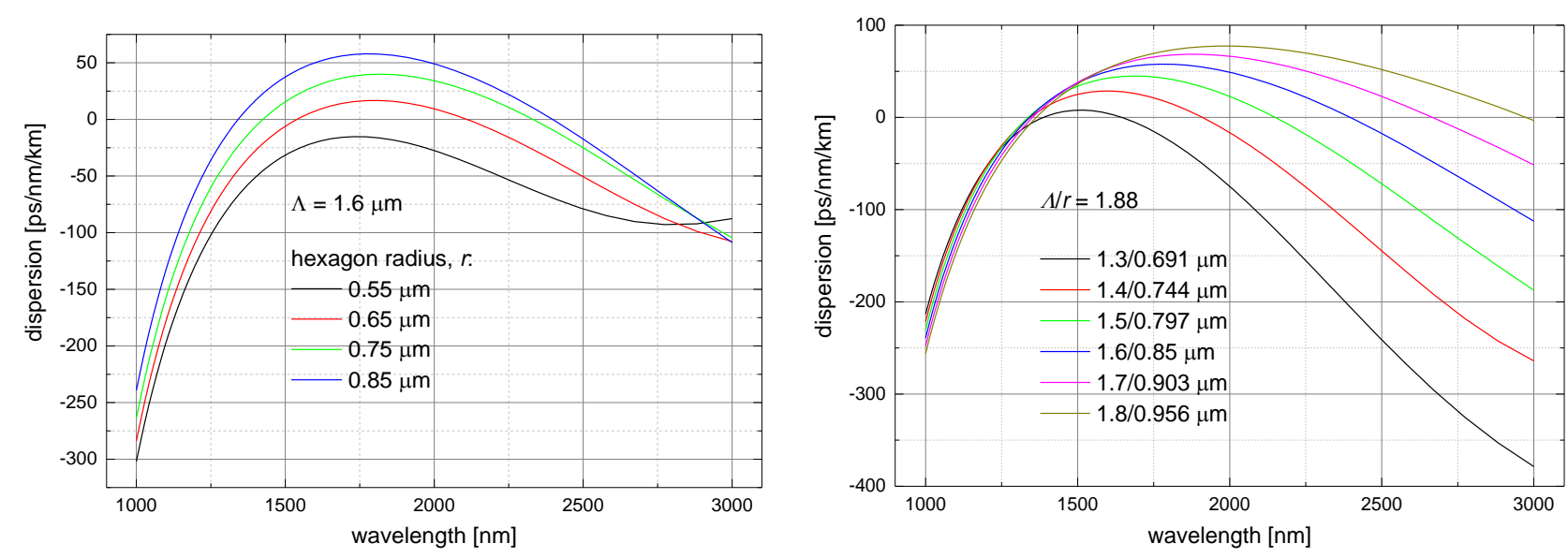

Fig. 2 Dispersion profiles of hexagonal type single-core all-solid PCF fiber at constant pitch of $1.6 \mu \mathrm{m}$ and changing hexagon radius (left) and at constant fill factor ensured by constant $\Lambda / r$ (right).

In Fig. 2(left), the single-core dispersion curves of the analyzed structures with constant pitch of $1.6 \mu \mathrm{m}$ and varying outer radius of the low index hexagons are presented. The results reveal that the lower $\Lambda / r$ of the structure, the higher measure of anomalous dispersion in our region of interest. However, in the case of 0.85 $\mu \mathrm{m}$ rod radius, the surface ratio of the low index areas in the lattice (fill factor) is around $85 \%$, which is the upper limit from point of view of technological feasibility. Therefore, in the next step we fixed the $A / r$ at this maximal value of 1.88 and the effect of the structural period was analyzed. The results in Fig. 2(right) show, that by increasing structural period, it is possible to increase the measure of the anomalous dispersion, which is accompanied by monotonic shift of the dispersion maxima towards the mid infrared region. If one focuses on the C-band, the dispersion is very similar for structural pitch values in range of $1.6-1.8 \mu \mathrm{m}$, with slight 
increase of the TOD measure with increasing $\Lambda$. It is disadvantageous from point of view of solitonic propagation. However, most importantly, the lower pitch value ensures shorter coupling length and shorter soliton compression length due to the stronger nonlinear interaction according to equations (1) - (3). Thus, finally, structural parameter combination of $1.6 / 0.85 \mu \mathrm{m}$ was chosen as optimum from point of view of anomalous dispersion and envisaged device length as the outcome of the optimization process. According to the numerical analysis, the selected structure has $13 \mathrm{~mm}$ coupling length at $1550 \mathrm{~nm}$. This represents weaker coupling than in the case of the previously published optimized air-glass PCF structure, which expressed $L_{C}$ $=3.7 \mathrm{~mm}$ at the same wavelength [16]. The main reason of the elongation of the coupling length is the larger structural pitch which was only $1.3 \mu \mathrm{m}$ in the case of the air-glass PCF. In the next step, the nonlinear dualcore propagation analysis was launched incorporating the wavelength dependences of the effective mode area, dispersion and coupling length.

The optimization of the excitation parameters, i.e. wavelength, pulse width and pulse energy, was performed in this framework in order to establish the soliton self-trapping process. The linear propagation characteristics changed significantly in comparison to the previously studied air-glass DC PCF structure. The measure of the anomalous dispersion decreased about 4 times and the coupling length increased also about 4 times in the C-band. Therefore, it is not possible to fulfill the condition to have comparable coupling length and soliton compression distance at the same excitation parameters (wavelength, pulse width, pulse energy) as was presented in the case of the air-glass PCF structure. However, this discrepancy is possible to overcome by optimization of excitation wavelength and pulse width. The coupling length $L_{\mathrm{C}}$ decreases rapidly with elongation of the wavelength, as it is shown in Fig 6. (right) in the case of three different dualcore fibers. Therefore, similar $L_{C}$ value is attainable in the case of the all-solid structure as in the case of the air-glass one by increasing the wavelength from $1550 \mathrm{~nm}$ to $1700 \mathrm{~nm}$. On the other hand, the impact of reduced anomalous dispersion can be compensated by decreasing the pulse width according to equation (1) in order to keep the soliton order at the same level as in the air-glass PCF case. However, it is necessary to consider, that the air-glass structure had lower pitch of $1.3 \mu \mathrm{m}$, therefore, the new structure with pitch of 1.6 $\mu \mathrm{m}$ expresses larger effective mode area and lower nonlinear parameter according to relation

$$
\gamma=2 \pi n_{2} / \lambda A_{e f f}
$$

where $n_{2}$ is nonlinear refractive index of the guiding PBG-08 glass equal to $4.3 \cdot 10^{-19} \mathrm{~m}^{2} / W$ [14], $\lambda$ is the wavelength and $A_{\text {eff }}$ is the effective mode area. Considering these differences, only moderate reduction of the pulse width is necessary in order to achieve similar soliton numbers $(\sim 4)$ in the case of the newly optimized all-solid PCF at the same pulse energy levels, i.e. 120 pJ. Thus, the combination of excitation pulse parameters of $1700 \mathrm{~nm}, 70 \mathrm{fs}$ and $120 \mathrm{pJ}$ results in soliton compression distance of $3.5 \mathrm{~mm}$ according to equation (3). This is equal to the value calculated in the case of the air-glass PCF structure under excitation by $1550 \mathrm{~nm}, 100 \mathrm{fs}, 120 \mathrm{pJ}$ pulses, balanced with the similar coupling length value in the both cases.

\section{Switching performance analysis by numerical simulation of the nonlinear dual-core propagation}

The above described estimation of the right pulse parameters was confirmed by the numerical simulations of the nonlinear propagation. From point of view of pulse self-trapping tendency, the best combination was $1700 \mathrm{~nm}$ and $70 \mathrm{fs}$ from parameter ranges of $1500-1700 \mathrm{~nm}$ and $70-150 \mathrm{fs}$. Despite the large amount of propagation maps created by sequentially increasing the input pulse energies up to $200 \mathrm{pJ}$ for different combinations of wavelengths and pulse widths, no conditions with clear self-trapping behavior were found. Numerical results for $1700 \mathrm{~nm}$ excitation pulse with $70 \mathrm{fs}$ width, for which promising switching performance was predicted, are presented in Fig. 3. The figure contains propagation maps as 2D spatial-time representation of the pulse intensity evolution with logarithmic color scale in $\mathrm{dB}$ units. The 4 maps represent the excited (left) and non-excited (right) core at $129 \mathrm{pJ}$ (top) and $141 \mathrm{pJ}$ (bottom) in-coupled excitation energy. The signature of the self-trapping behavior is observable on the maps in the area of $5-10 \mathrm{~mm}$ of 
propagation distance. At the lower energy, the cross (non-excited) core dominates, and at higher energy, the bar (excited) one, as the shorter soliton compression distance upon energy increase causes trapping of the soliton already in the excited core. However, the self-trapping process is not maintained beyond $10 \mathrm{~mm}$ due to the losses caused by intensive dispersive wave generation (DWG) process. The DWG is recognizable by the smooth straight lines extending towards the positive half of the time domain map, representing shorter wavelengths. Thus, the low level of anomalous dispersion in comparison to the model air-glass PCF structure, combined with more pronounced compression and nonlinear behavior, does not support the highly effective self-trapping process. The soliton fission process evolves sequentially and, in the later propagation phase, causes multiple solitonic features (represented by red colored lines), which are further disturbed by the coupling oscillations between the cores. Nevertheless, after the soliton compression distance, relatively long stable line of the compressed most intensive feature occurs at the lower excitation energy in the cross core with peak at $8 \mathrm{~mm}$. In contrast, the excited core dominates at higher excitation energy around the same area of propagation distance, accompanied by significantly weaker field intensities in the non-excited core.
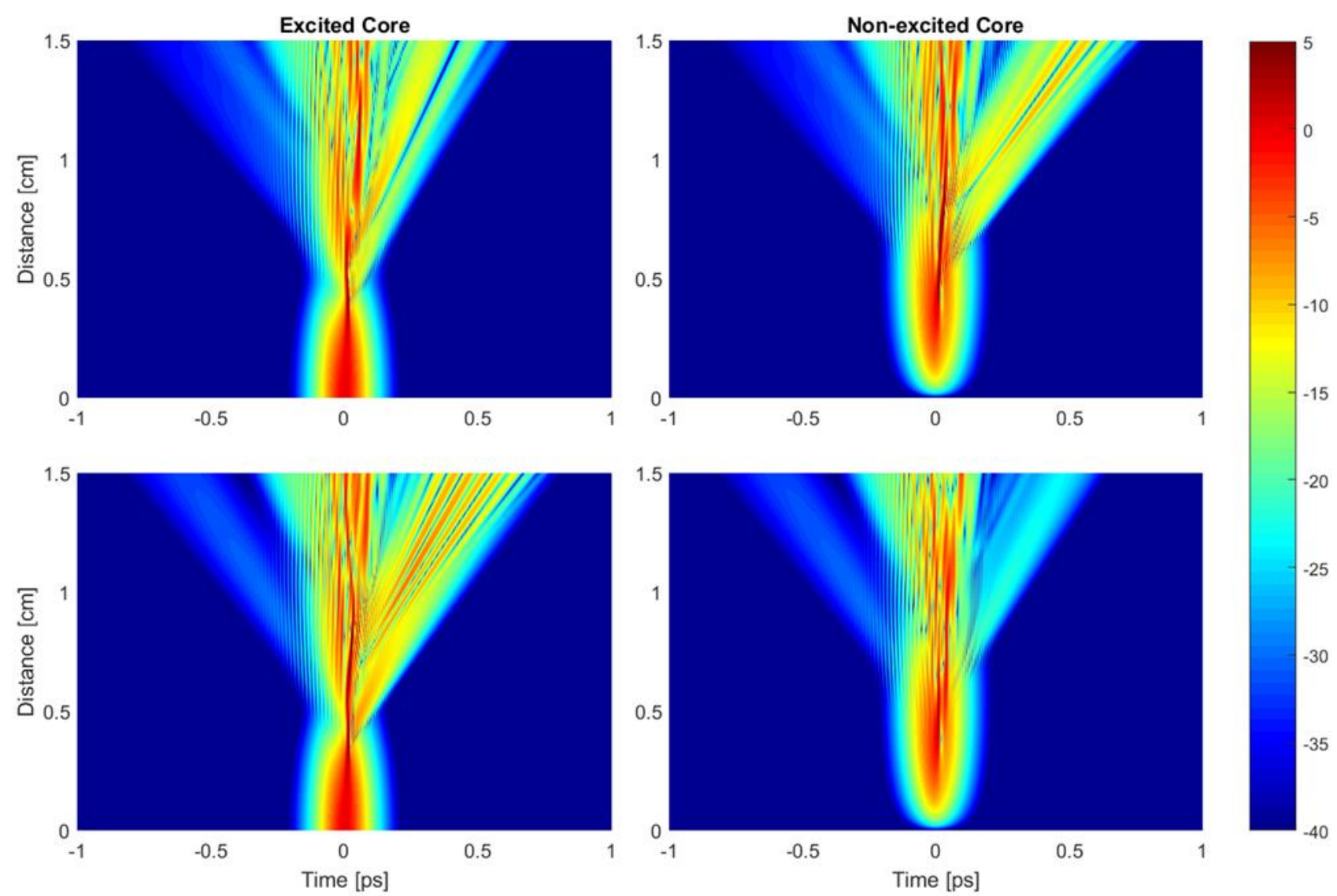

Fig. 3 Best found switching performance considering optimized all-solid PCF structure under excitation by $1700 \mathrm{~nm}$, $70 \mathrm{fs}$ pulses, which expresses switching length of $9.6 \mathrm{~mm}$. Time domain evolution of the field intensity in the excited (left) and non-excited (right) core at $129 \mathrm{pJ}$ (top) and $141 \mathrm{pJ}$ (bottom) pulse energies.

In order to evaluate quantitatively the switching performance, the propagation maps were integrated line by line and the spatial evolution of the field energy was tracked in the all four presented cases. Next, the dependence of the dual-core extinction ratio on the discretized propagation distance $z_{\mathrm{i}}$ in $\mathrm{dB}$ units was calculated according to formula

$$
E R\left(E_{1,2}, z_{i}\right)=10 \log \left(\frac{E_{\text {bar }}\left(E_{1,2}, z_{i}\right)}{E_{\text {cross }}\left(E_{1,2}, z_{i}\right)}\right)
$$

where $E_{1}, E_{2}$ are the lower and higher in-coupled switching energies, respectively and $E_{\text {bar }}, E_{\text {cross }}$ are the integrated field energies in the bar and the cross core. Finally, the $z_{\mathrm{i}}$ dependence of switching contrast is calculated by subtraction of the extinction ratios at the two energy levels $\operatorname{SC}\left(z_{\mathrm{i}}\right)=E R\left(E_{2}, z_{\mathrm{i}}\right)-E R\left(E_{1}, z_{\mathrm{i}}\right)$. 
According to the performed switching analysis, the highest value of $S C\left(z_{\mathrm{i}}\right)$ was found at $8 \mathrm{~mm}$ of propagation distance. Thereafter, we will call it switching length $L_{\mathrm{s}}$.

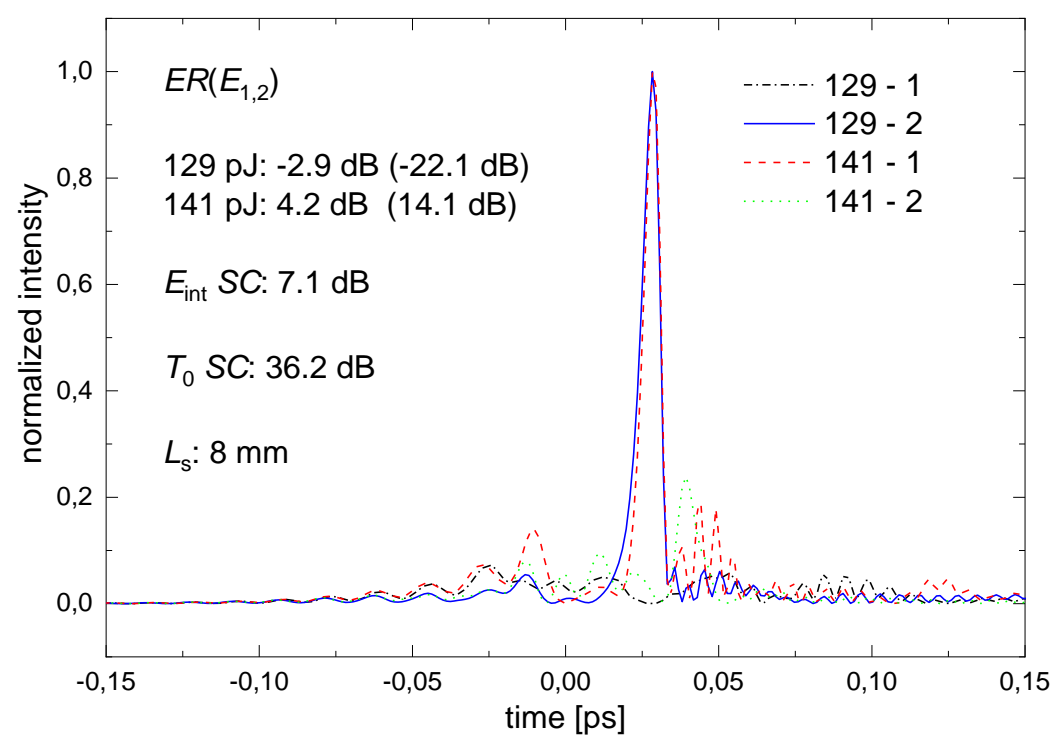

Fig. 4 Time dependence of the field intensity envelope at the switching length $(8 \mathrm{~mm})$ in the both cores and at the both switching energies. All-solid DC PCF excited with $1700 \mathrm{~nm}, 70$ fs pulses.

Fig. 4 depicts the temporal envelopes of field intensity at the switching length $L_{\mathrm{s}}$ taken from the 4 propagation maps in Fig. 3, but already on linear scale of intensity. The curves are normalized separately for the both input energies in order to have the peak intensity in the dominant core always at value 1 . The outcome of the overall analysis is rather low switching contrast of $7.1 \mathrm{~dB}$, even in this case of the best combination of excitation wavelength and pulse width. In the case of the previously published optimized airglass PCF structure, the switching contrast was at the level of $12 \mathrm{~dB}$, so the optimized all-solid PCF structure supports only moderate switching with nearly two times lower contrast. The switching contrast increases significantly, when we consider the field energy just in the time window of the short self-trapped solitonic pulses. This evaluation approach is also appropriate from the application point of view, where the switched pulses will interact with timely synchronized short optical signals. In this case, the pre- and post-pulses will have no effect on the interaction. Similarly, only the time window of the main pulse envelope contributes significantly to a nonlinear signal applying the switched pulses for any type of subsequent nonlinear interaction.

Therefore, we fitted the time envelopes $I(t)$ of the both main solitons in the dominant core at the lower and higher energy with $\operatorname{sech}^{2}$ function. The resulted curves $F_{1,2}(\mathrm{t})$ were used to calculate the integral

$$
E_{j}^{i}=\int F_{j}(t) I_{j}^{i}(t) d t
$$

where the upper index denotes the core and the lower one the energy level. The obtained four values of the temporally localized energy were used to calculate the extinction ratios and the switching contrast similarly to the integral energy evaluation method and resulted in $S C_{\mathrm{T}}=36.2 \mathrm{~dB}$. This value is a significant improvement in comparison to the integral energy switching contrast and it originates from the character of the temporal intensity distributions in the dominant and non-dominant cores. In the case of the both switching energies, most of the field intensity is beyond the time window of the main soliton in the nondominant cores. The obtained temporally localized switching contrast is even slightly higher, than in the case of the air-glass PCF, where contrast of $34.8 \mathrm{~dB}$ was achieved. Thus, the best-found switching performance in the case of the all-solid DC PCF promises the feasibility of an effective all-optical switching device by this technological approach. On the other hand, it requires further improvement of the fiber design because it does not support the effective soliton self-trapping. Therefore, despite the optimization of the structure and 
the excitation parameters, only $6 \mathrm{~dB}$ integrated energy switching contrast was achieved. This value is too low from application point of view, when simple detectors with time integration character are used for the electro-optical conversion of the switched signal.
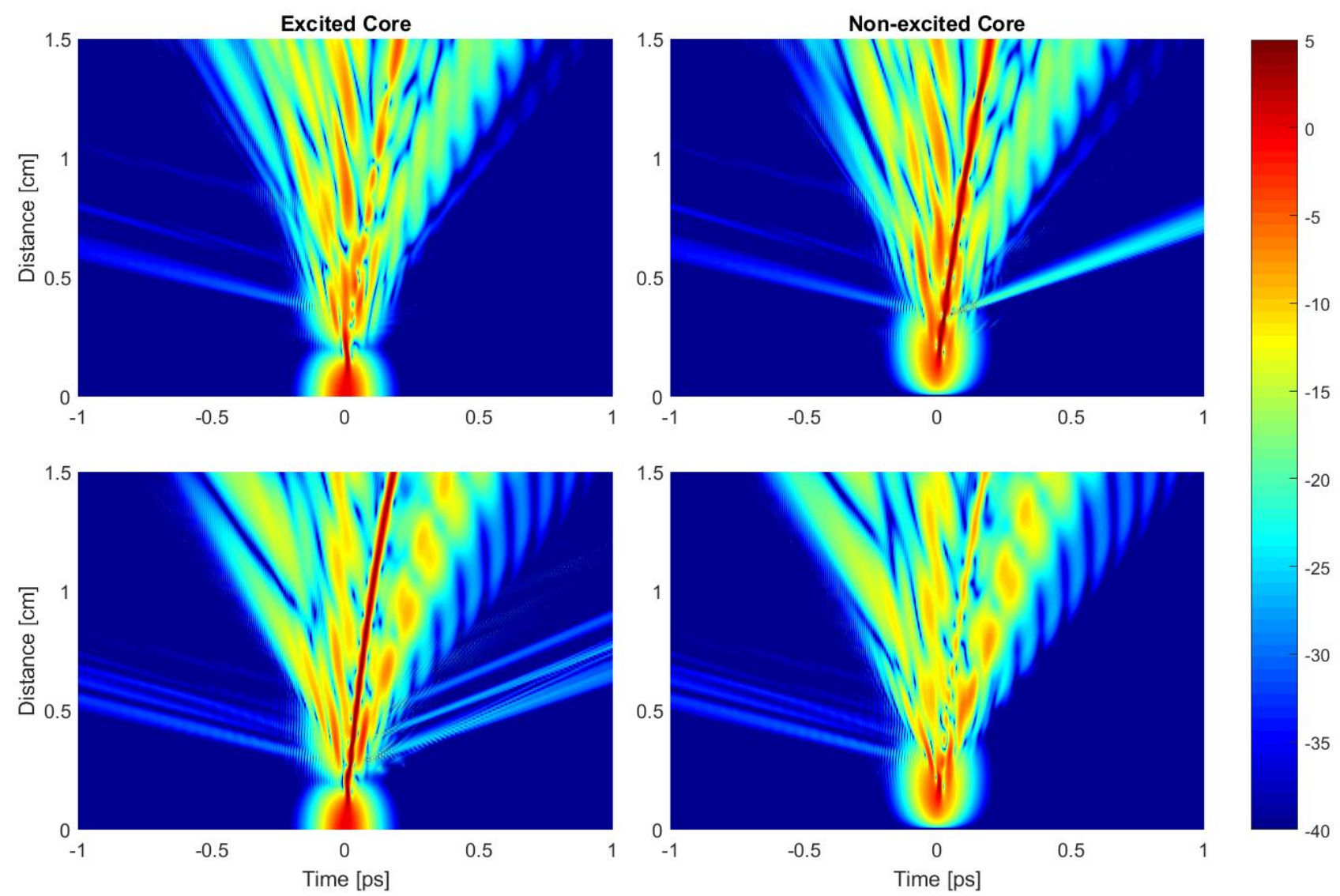

Fig. 5 Self-trapped soliton switching performance considering air-glass DC PCF under excitation by $1700 \mathrm{~nm}, 70 \mathrm{fs}$ pulses, which expresses switching distance of $7 \mathrm{~mm}$. Time domain evolution of the field intensity in the excited (left) and nonexcited (right) core at $231 \mathrm{pJ}$ (top) and $241 \mathrm{pJ}$ (bottom) pulse energies.

In the next step, we returned to the previously analyzed air-glass DC PCF structure in order to verify its applicability when changing the excitation wavelength and the pulse width. It is similar hexagonal lattice PCF with structural parameters $\Lambda=1.3 \mu \mathrm{m}, r=0.5 \mu \mathrm{m}$, however it contains air circles instead of low index glass hexagons. The single-core dispersion profiles of the studied all-solid and the air-glass DC PCFs are presented in Fig. 6 and express significantly higher measure of the anomalous dispersion in the latter case. On the other hand, the both profiles have the dispersion maxima in the same area of $1750-1800 \mathrm{~nm}$. Therefore, one can expect improved switching performance in the case of the air-glass structure at $1700 \mathrm{~nm}$ as obtained in the case of the to the all-solid structure, which provides the best switching results at this excitation wavelength. The earlier published study of the air-glass DC PCF was focused only on the C-band at pulse width of $100 \mathrm{fs}$ [16], now we extended it for the pulse parameters $1700 \mathrm{~nm}, 70 \mathrm{fs}$. The pulse width was decreased to reduce the soliton order, because the longer wavelength causes again shorter $L_{\mathrm{C}}$, which requires shorter $z_{\text {comp, }}$ as well. By this approach, we tested the parameters, which supported the best switching performance considering the new all-solid DC PCF structure. Again, at first the lowest energies which ensure the self-trapping in the cross and the bar core were identified. Then, the switching length leading to the best integral energy switching contrast was determined. The results are presented in Fig. 5 in the form of 4 propagation maps at the identified switching energies of $231 \mathrm{pJ}$ (top row) and $241 \mathrm{pJ}$ (bottom row). Despite the change of wavelength and pulse width, it expresses very similar switching performance in comparison to the previous study of the optimized air-glass PCF. Clear soliton self-trapping effect was found in the case of the both switching energies. The self-trapping is preserved up to the end of the simulated propagation distance in convincing manner. The switching parameters are even better than in the case of the 
previously published combination of $1550 \mathrm{~nm}$ and $100 \mathrm{fs}$. The switching length was reduced from $11 \mathrm{~mm}$ to $7 \mathrm{~mm}$, the integral energy switching contrast $S C_{\mathrm{E}}$ increased from $11.8 \mathrm{~dB}$ to $13.4 \mathrm{~dB}$ and the temporally localized switching contrast $S C_{\mathrm{T}}$ from $34.8 \mathrm{~dB}$ to $38.4 \mathrm{~dB}$. The soliton number was preserved to be 4 despite the change of the both excitation parameters. However, the soliton compression distance was significantly reduced, because it should be balanced with the shortened coupling length $\left(L_{\mathrm{C}}=2 \mathrm{~mm}\right)$. On the other side, the change of excitation parameters caused increase of the switching energies by factor 2 . The improved switching performance originates mainly from the higher value of anomalous dispersion and lower TOD according to the dispersion profile (green curve in Fig. 6). Most importantly, at the both combinations of wavelength and pulse width, the pulse compression is moderate and lower measure of dispersive waves is generated than in the case of the all-solid PCF (Fig. 3). Thus, the modeled air-glass PCF structure is advantageous from the point of view of all-optical switching and it is robust against alteration of the excitation wavelength or the pulse width. In order to establish such advantageous structure using the all-solid approach, the differences between the dispersion profiles of considered DC architectures were compared and structural changes suggested.
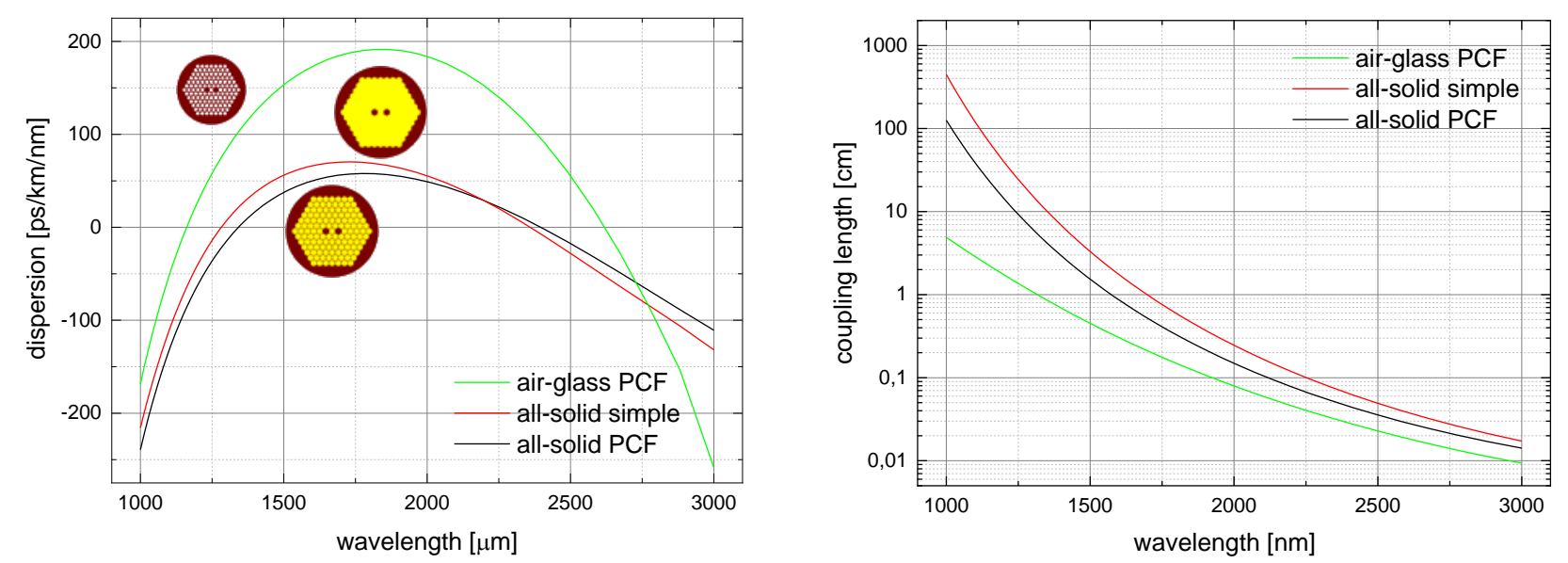

Fig. 6 Dispersion profile of the single-core waveguide with the corresponding picture of the fiber cross sections (left) and coupling length characteristics (right) in the case of optimized structure of air-glass PCF, all-solid PCF and simple all-solid dual-core fibers.

Fig. 6 illustrates the dispersion aspect of the large scope optimization process, displaying the single-core dispersion profiles of the optimized air-glass and the all-solid DC PCF. Regarding the measure of anomalous dispersion, the difference between the two DC PCF architectures is significant. The anomalous dispersion range is nearly two times broader in the case of the air-glass PCF and its value is 4 times larger, not only in the C-band, but also at the dispersion maxima. In order to eliminate such large difference, the fill factor of the dual-core cladding needs to be maximized, as motivated by the trend presented in Fig. 2(left). However, the structure with maximal fill factor is actually a homogeneous low index area without photonic structure, i.e. without high index bridges. This change of the strategy had advantageous effect on the dispersion profile, which is presented in Fig. 6 with red curve. The profile corresponds to the homogeneous cladding structure with the unchanged pitch of $1.6 \mu \mathrm{m}$. The value of the anomalous dispersion increased by factor 2 in the Cband in comparison to the all-solid PCF structure, which is advantageous from point of view of solitonic propagation. The only drawback of the homogeneous cladding is the reduced coupling, resulting in about two times longer coupling length of $24 \mathrm{~mm}$ at $1550 \mathrm{~nm}$ [22]; this prevents the demonstration of sub-cm coupling device in spectral range of our interest $(1400 \mathrm{~nm}-1700 \mathrm{~nm})$.

In order to identify the influence of the new structure design on the switching performance, the nonlinear simulation analysis was performed. At first, the common excitation parameters, $1700 \mathrm{~nm}$ and $70 \mathrm{fs}$, were used in order to directly compare the obtained results with the previously presented ones in the case of the all-solid and air-glass PCF architectures. The $1700 \mathrm{~nm}$ excitation wavelength is also in this case very close to the dispersion maxima and its combination with pulse width $70 \mathrm{fs}$ results in switching energies determining soliton order again at the level of 4 . Fig. 7 compares the switching performance in the case of the air-glass 
PCF structure (left) from propagation maps in Fig. 5 and in the case of the homogeneous cladding all-solid one (right). According to the comparison, the new structure supports similar switching contrasts at the level of $12.1 \mathrm{~dB}$ regarding the integral energy and $32.5 \mathrm{~dB}$ regarding the temporally localized one. The switching length was elongated from $7 \mathrm{~mm}$ to $16 \mathrm{~mm}$ due to the lower coupling in the case of the homogeneous cladding structure with pitch of $1.6 \mu \mathrm{m}$. On the other hand, the larger coupling length requires longer soliton compression distance $z_{\text {comp }}$, which reduces the pulse energy requirement. According to equations (2-4), the lower pulse energy due to the decreasing peak power increases the $z_{\text {comp }}$ because of the reduced soliton order. Therefore, the switching energies in the case of the new structure are 3 times lower in comparison to the airglass PCF at the same excitation parameters. It is worth mentioning, that the energy pair $67-73 \mathrm{pJ}$ presented in this work is up to now the lowest energy level supporting the self-trapped soliton switching. It is already in sub-100 $\mathrm{pJ}$ in-coupled energy range, which is an important progress from application point of view.
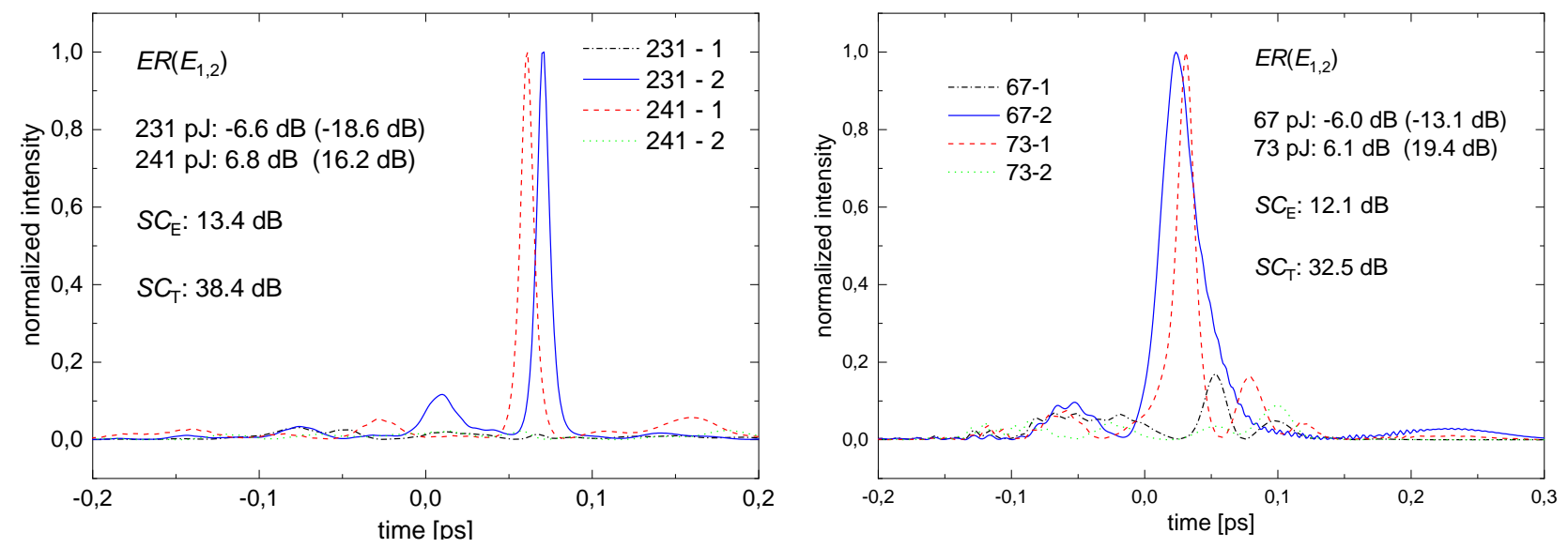

Fig. 7 Time dependence of the field intensity envelope at the switching length in the both cores and at the both switching energies. Air-glass PCF (left) and all-solid homogeneous cladding (right) dual-core fiber excited with $1700 \mathrm{~nm}, 70 \mathrm{fs}$ pulses.

After the confirmation of the applicability of the new simpler dual-core structure under excitation by 1700 $\mathrm{nm}, 70 \mathrm{fs}$ pulses, the complex switching performance analysis process was launched again, as for the case of the all-solid PCF. The analysis was performed by changing both the wavelength and the pulse width in ranges of $1400-1800 \mathrm{~nm}$ and $75-150 \mathrm{fs}$, respectively, looking for the best combination from point of view of switching contrast. The detailed report about this analysis has been already published [22], here we just summarize it. The combination of $1500 \mathrm{~nm}$ excitation wavelength and $75 \mathrm{fs}$ pulse width resulted in the best integrated energy switching contrast to be $17.2 \mathrm{~dB}$ at $43 \mathrm{~mm}$ switching length $L_{\mathrm{S}}$. The temporally localized switching contrast increased already over $40 \mathrm{~dB}$ level and reached value of $46.3 \mathrm{~dB}$. Due to the strong wavelength dependence of the coupling length, $L_{C}$ was elongated again by a factor of two in relation to 1700 $\mathrm{nm}$ and reaches value of $33 \mathrm{~mm}$. However, the further elongation of $L_{\mathrm{C}}$ and corresponding $L_{\mathrm{S}}$ relaxed the switching energy requirement even more. Switching energies were identified at the level of $20 \mathrm{pJ}$ in the case of excitation by $1500 \mathrm{~nm}, 75$ fs pulses. Such low energy requirement promises realization of the self-trapped soliton switching concept using sub-100 pJ femtosecond sources. It means that simple fiber oscillators without any amplification stage can be used. This approach was not possible in the case of the previously published air-glass PCF, which required about $120 \mathrm{pJ}$ in-coupled energy. Moreover, the optimal wavelength is close to the C-band and the pulse width is comparable to the values typical for Er-doped fiber oscillators available on the market. 

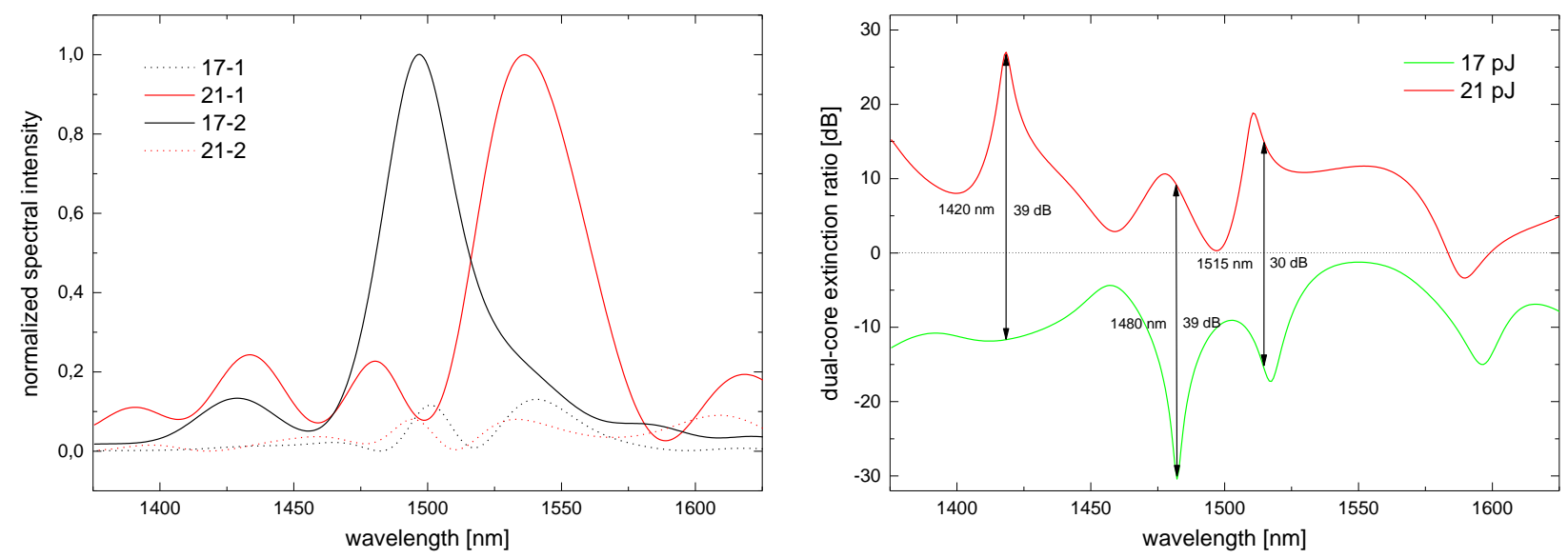

Fig. 8 Wavelength dependence of: (left) field intensity envelope at the switching length of $43 \mathrm{~mm}$ in the both cores and at the both switching energies; (right) dual-core extinction ratio of the spectral intensities. Homogeneous cladding high index contrast dual-core fiber excited with $1500 \mathrm{~nm}, 75 \mathrm{fs}$ pulses.

Fig. 8 shows the spectral domain representation of the best switching performance under excitation of the simple all-solid structure with pulses of $1500 \mathrm{~nm}$ wavelength and $75 \mathrm{fs}$ temporal width. The nonlinear spectral broadening is significant and exhibits output bandwidth beyond $200 \mathrm{~nm}$, however, in the left graph, only the central area with applicable spectral intensities from point of view of optical signal switching is depicted. The spectral profiles are normalized to 1 for each of the two switching energies, however the intensity relation between the two cores is preserved in the case of the both energies. In the right graph, the wavelength dependence of the dual-core extinction ratio is presented. It expresses broadband and uniform character of the self-trapped soliton switching concept. In the whole spectral range, the same switching performance from the non-excited to the excited core is predicted with increasing pulse energy between 17 and $21 \mathrm{pJ}$. The switching contrast related to dual-core extinction ratio of the spectral intensities $S C_{\mathrm{S}}$ exceeds the level of $10 \mathrm{~dB}$ for most of wavelengths. Typical $S C_{\mathrm{S}}$ values are around $20 \mathrm{~dB}$. Moreover, there are some peaks with exceptional switching contrast at wavelengths $1420 \mathrm{~nm}, 1480 \mathrm{~nm}$ and $1515 \mathrm{~nm}$, expressing $S C_{\mathrm{S}}$ values of $39 \mathrm{~dB}, 39 \mathrm{~dB}$ and $30 \mathrm{~dB}$, respectively. It suggests spectrally resolved multichannel application potential of the studied novel switching concept. The best experimentally achieved switching contrast studied in the spectral domain was $15 \mathrm{~dB}$ using an air-glass dual-core PCF with switching energies 20 and 50 $\mathrm{nJ}$ (measured before the in-coupling optics) [14]. Because the self-trapped solitonic propagation has not been ensured there, the spectral domain representation did not reflect such uniform switching regime as presented in Fig. 8. Further, the nonlinear refraction index of the used multicomponent glass has been only at the level of the standard silica glass, which did not allow to work in the sub-nJ range of switching energies. Thus, the numerical study of the novel switching concept for relatively easily fabricable all-solid DC fiber, which is proposed in this paper, promises significant improvement of previous experimental results.

The experimental setup to test solitonic switching in all-solid DC fiber will be the same as the one used for air-glass PCF. A detailed description of it is reported in Curilla et al. manuscript [15]. The input pulse wavelength will be kept around $1500 \mathrm{~nm}$ and its temporal width around $75 \mathrm{fs}$, in order to achieve the same values of SC values we have predicted in the frame of this paper. The fiber will be then shortened sequentially by few millimeter steps the and whole output field registration process will be repeated at every fiber length. This approach, called cut-back method, will help us to identify the most promising switching performances in the new all-solid DC fiber and, hopefully, to have the first verification of solitonic switching in dual-core fibers.

\section{Summary}

An extensive numerical simulation study devoted to all-optical switching based on soliton self-trapping process in highly nonlinear multicomponent glass dual-core optical fibers was presented in this paper. The large scope optimization process of the dual-core fiber structure included air-glass PCF, all-solid PCF and 
simple all-solid alternatives. Following our previous work, where the air-glass PCF geometry was optimized, here we focused on the all-solid PCF geometry variations by means of numerical calculation of the dispersion and coupling dispersion profiles. The optimized high index contrast all-solid PCF did not support better switching performance, than the previously published air-glass PCF. Therefore, in a following step, a simple dual-core fiber structure with homogeneous cladding was considered in the framework of nonlinear propagation simulations and switching performance analysis. Comparing the three different structural alternatives, the lowest switching energies at common excitation parameters (1700 nm and $70 \mathrm{fs}$ pulses) were predicted for the simple DC structure. The further optimization of the excitation wavelength and pulse width resulted in even lower switching energies at simultaneous improvement of the switching contrasts at combination of $1500 \mathrm{~nm}$ and $75 \mathrm{fs}$. The integral energy and the temporally localized switching contrasts were identified to be $17.2 \mathrm{~dB}$ and $46.3 \mathrm{~dB}$, respectively. Up to now, these are the best values achieved by our approach. Moreover, the predicted in-coupled switching energies are only at the level of $20 \mathrm{pJ}$, representing a significant progress in comparison to similar works. Additionally, spectral aspect of this advantageous switching performance was presented, revealing its broadband and uniform character with switching contrasts exceeding $30 \mathrm{~dB}$ at three different wavelengths relevant to optical communication technology. Finally, we can state, that the suggested approach is promising also from application point of view, considering relatively easily fabricable simple cladding structure from realistic glasses.

\section{Acknowledgement}

This work was supported by National Science Centre, Poland [project No. 2016/23/P/ST7/02233 under POLONEZ program which has received funding from the European Union's Horizon 2020 research and innovation program under the Marie Skłodowska-Curie grant agreement No 665778], by Slovak R\&D Agency under the contracts No. SK-AT-2017-0026, APVV-14-0716 and by Austrian Agency OeAD under contract number SK 02_2018.

\section{References}

[1] A. Betlej, S. Suntsov, K.G. Makris, L. Jankovic, D.N. Christodoulides, G.I. Stegeman, J. Fini, R.T. Bise, D. J. DiGiovanni : All-optical switching and multifrequency generation in a dual-core photonic crystal fiber, Opt. Lett. 31 (2006) 1480-1482

[2] A.G. Coelho, M.B.C. Costa, A.C. Ferreira, M.G. da Silva, M.L. Lyra, A.S.B. Sombra; Realization of AllOptical Logic Gates in a Triangular Triple-Core Photonic Crystal Fiber, Journal of Lightwave Technology 31 (2013) 731-739

[3] C.C. Lee, P.K.A. Wai, H.Y. Tam, L. Xu, C. Wu: 10-Gb/sWavelength Transparent Optically Controlled Buffer Using Photonic-Crystal-Fiber-Based Nonlinear Optical Loop Mirror, IEEE Photon. Technol. Lett. 19 (2007) 898-900

[4] J. Hu, Y.-P. Huang, P. Kumar: Self-stabilized quantum optical Fredkin gate, Opt. Lett. 38 (2013) 522

[5] S. Trillo, S. Wabnitz, E. M. Wright, G.I. Stegeman: Soliton switching in fiber nonlinear directional couplers, Opt. Lett. 13 (1988) 672

[6] Y. Wang, W. Wang, "Nonlinear Optical Pulse Coupling Dynamics," IEEE J. Lightwave Technol. 24 (2006) 2458.

[7] A.H.Arnous, S.A.Mahmood, M.Younis, Dynamics of optical solitons in dual-core fibers via two integration schemes, Superlattices and Microstructures 106 (2017) 156-162

[8] S. R. Friberg, A. M. Weiner, Y. Silberberg, B. G. Sfez, P. S. Smith: Femotosecond switching in a dualcore-fiber nonlinear coupler, Opt. Lett. 13 (1988) 904-906

[9] Y.S. Kivshar: Switching dynamics of solitons in fiber directional couplers, Opt. Lett. 18 (1993) 7-9

[10] J. H. Li, K. S. Chiang, K. W. Chow: Switching of ultrashort pulses in nonlinear high-birefringence twocore optical fibers, Optics Communications 318 (2014) 11-16 
[11] K. Nithyanandan, R. Vasantha Jayakantha Raja, K. Porsezian: Modulational instability in a twin-core fiber with the effect of saturable nonlinear response and coupling coefficient dispersion, Phys. Rev. A 87 (2013) 043805

[12] A.K. Sarma: Soliton Switching in a Highly Nonlinear Dual-Core Holey Fiber Coupler, Japanese Journal of Applied Physics 47 (2008) 5493-5495

[13] S. Pricking, M. Vieweg, H. Giessen: Influence of retarded response on an ultrafast nonlinear optofluidic fiber coupler, Opt. Express 19 (2011) 21673-21679

[14] P. Stajanca, D. Pysz, G. Andriukaitis, T. Balciunas, G. Fan, A. Baltuska, I. Bugar, Ultrafast multiwavelength switch based on dynamics of spectrally-shifted solitons in a dual-core photonic crystal fiber, Opt. Express. 22 (2014) 31092-31101

[15] L. Curilla, I. Astrauskas, A. Pugzlys, P. Stajanca, D. Pysz, F. Uherek, A. Baltuska, I. Bugar, Nonlinear performance of asymmetric coupler based on dual-core photonic crystal fiber: Towards sub-nanojoule solitonic ultrafast all-optical switching, Opt. Fiber Technol. 42 (2018) 39-49

[16] P. Stajanca, I. Bugar, Nonlinear ultrafast switching based on soliton self-trapping in dual-core photonic crystal fibre, Laser Phys. Lett. 13 (2016) 116-201

[17] M. Klimczak, B. Siwicki, P. Skibiński, D. Pysz, R. Stępień, A. Heidt, C. Radzewicz, R. Buczyński, Coherent supercontinuum generation up to $2.3 \mu \mathrm{m}$ in all-solid soft-glass photonic crystal fibers with flat allnormal dispersion, Opt. Express. 22 (2014) 18824-18832

[18] J. Cimek, R. Stępień, G. Stępniewski, B. Siwicki, P. Stafiej, M. Klimczak, D. Pysz, R. Buczyński, High contrast glasses for all-solid fibers fabrication, Opt. Mater. (Amst). 62 (2016) 159-163

[19] L. Wang, D. He, S. Feng, C. Yu, L. Hu, J. Qiu, D. Chen; Yb/Er co-doped phosphate all-solid singlemode photonic crystal fiber, Scientific Reports 4, 6139 (2014)

[20] S. R. Papior, J. Weirich, M. M. Johansen, C. Jakobsen, M. Michieletto, M. Triches, T. Kristensen, A. S. Olesen, C. Petersen, T. V. Andersen, M. D. Maack, T. T. Alkeskjold; Photonic crystal fiber technology for high-performance all-fiber monolithic ultrafast fiber amplifiers, Proceedings of SPIE 10512 (2018) 10512-37

[21] J. Zhao, Z. Wang, Y. Liu, B. Liu, J. Zhao, Z. Wang, Y. Liu, B Liu, Switchable-multi-wavelength fiber laser based on dual-core all-solid photonic bandgap fiber; Frontiers of Optoelectronics in China 3 (2010) $283-288$

[22] M. Longobucco, J. Cimek, L. Curilla, D. Pysz, R. Buczynski, I. Bugar, All-optical switching based on soliton self-trapping in dual-core high-contrast optical fibre, Optical Fiber Technology 51 (2019) 48-58

[23] Agrawal G P 2001 Applications of Nonlinear Fibre Optics (New York: Academic)

[24] J. Herrmann, U. Griebner, N. Zhavoronkov, A. Husakou, D. Nickel, J.C. Knight, W.J. Wadsworth, P.S. Russell, G. Korn, Experimental evidence for supercontinuum generation by fission of higher-order solitons in photonic fibers, Phys. Rev. Lett. 88 (2002) 1739011-1739014 